Ophthalmologe 2020 $\cdot 117: 671-676$ https://doi.org/10.1007/s00347-019-00983-9 Online publiziert: 18. Oktober 2019

(c) Der/die Autor(en) 2019

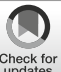

\section{S. Irle ${ }^{1}$ (D) $\cdot$ E. Msigomba $^{2} \cdot$ K. Paust $^{3}$}

${ }^{1}$ Augenklinik-Walsum GmbH, Duisburg, Deutschland

${ }^{2}$ Ilembula Lutheran Hospital, Ilembula, Tansania

${ }^{3}$ Gemeinschaftspraxis Bonner Augen, Bonn, Deutschland

\title{
Sumbawanga Augencamp follow-up Studie 2019
}

\section{Hintergrund}

Die World Health Organization (WHO) schätzt, dass weltweit ca. 18 Mio. Menschen an einer (beidseitigen) Katarakt erblindet sind („blinding cataract“). Die Katarakt ist mit $48 \%$ die Hauptursache für behandelbare Erblindung.

Während sich die Kataraktchirurgie zur Behandlung des grauen Stars in den westlichen Industrienationen $\mathrm{zu}$ einem der erfolgreichsten Operationsverfahren in der Medizin überhaupt entwickelt hat und Menschen auch in hohem Lebensalter eine exzellente Sehqualität ermöglicht, steigt in weniger entwickelten Ländern die Zahl der Kataraktblinden kontinuierlich. Diese Entwicklung kommt nicht unerwartet [16] und hängt wesentlich mit dem Anstieg der Zahl älterer Menschen weltweit zusammen und das, obwohl es erhebliche Anstrengungen gibt, vermeidbare Blindheit wirkungsvoll $\mathrm{zu}$ bekämpfen [20].

Nach wie vor ist in Afrika 1 Augenarzt für ca. 1.000.000 Menschen verantwortlich, die „cataract surgical rate“ (CSR), also die Zahl der Operationen pro Jahr pro 1 Mio. Menschen, liegt im südlichen Afrika (Sub-Sahara-Afrika [SSA]) bei unter 500 , in Tansania 2010 bei 518 Operationen [3]. Die CSR sollte zwischen 2000 bis 5000 Eingriffen auf 1 Mio. Menschen betragen, um Kataraktblindheit adäquat behandeln zu können $[2,11]$.

Programme zur Blindheitsverhütung bestehen mancherorts schon sehr lange. Seit Mitte der 90er-Jahre gibt es nachhaltige Strukturen, die dennoch an Effektivität $\mathrm{zu}$ wünschen übrig lassen [11].
Sumbawanga, eine 150.000-Einwohner-Stadt, liegt im Südwesten Tansanias und ist Hauptstadt der Region Rukwa. Die augenchirurgische Versorgung wird dort seit knapp 10 Jahren von einem einzelnen Kataraktchirurgen (Assistent Medical Officer [AMO], nicht universitärer staatlich anerkannter Ausbildungsberuf) für die gesamte Rukwa-Region mit etwa 1,5 Mio. Menschen sichergestellt.

Um die Versorgung zu verbessern, hat das Deutsche Komitee zur Verhütung von Blindheit (DKVB) seit $2017 \mathrm{Au}-$ gencamps eingerichtet: Zweimal jährlich fliegt ein Team aus mehreren Ärzten und medizinischem Fachpersonal nach Sumbawanga, um in einem jeweils 2-wöchigen Einsatz Patienten zu untersuchen und $\mathrm{zu}$ operieren. Pro Einsatz werden 800 bis 1000 Patienten untersucht und etwa 200 Operationen durchgeführt Tendenz steigend. Die Patienten kommen aus der gesamten Rukwa-Region, im Einzelfall über Entfernungen von mehreren 100 Kilometern angereist. Die Bedingungen in solchen Einsätzen mit Augencamp-Charakter sind naturgemäß schlechter als in einer etablierten Klinikstruktur [2]. Dennoch scheint es nach dem nunmehr vierten Camp in Sumbawanga angemessen, sich mit der Qualität der geleisteten Arbeit auseinanderzusetzen, zumal die Weltgesundheitsorganisation (WHO) sehr klare Vorgaben für die Ergebnisqualität in der Kataraktchirurgie formuliert [20].

Vielleicht ist es eine Schwäche von Hilfseinätzen, sich mehr auf die Zahl der Untersuchungen und Eingriffe zu konzentrieren als auf die Qualität der Ergebnisse. Das ist bedauerlich, zumal es nicht neu ist, dass Qualität eine gleicher- maßen hohe Aufmerksamkeit verdient wie Quantität. Speziell aus der SSA-Region gibt es keine veröffentlichten Studien über an Augenkliniken durchgeführte Kataraktserien, bei denen die Qualitätsziele der WHO erreicht wurden. Diese Tatsache hat dazu geführt, dass in den betroffenen Ländern - auch in Tansania - eine erhöhte Aufmerksamkeit für die Bedeutung der Qualität in der Kataraktchirurgie im öffentlichen Gesundheitswesen entstanden ist $[1,22]$.

In diesem Sinne stellen die Auswertung und Darstellung dieser zweifelsohne ernüchternden Daten den Beginn eines kontinuierlichen Ergebnismonitorings dar mit dem Ziel, auch unter „einfachen“ Bedingungen die eigene Ergebnisqualität $\mathrm{zu}$ dokumentieren, $\mathrm{zu}$ analysieren und Verbesserungspotenzial für die Zukunft $\mathrm{zu}$ evaluieren. Ergebnisqualität ist einer der wesentlichen Faktoren in einer „patientenfreundlichen“ Prozesskette, die das Wohl des Patienten im Blick hat [21]. Susan Lewallen hat bereits vor Jahren beschriebenen, dass es gut durchdachter, qualitativ hochwertiger und langfristig geplanter Programme bedarf, um die Cataract-surgical-Rate $\mathrm{zu}$ steigern [11, 22].

\section{Material und Methoden}

Das vierte Sumbawanga-Augencamp des Deutschen Komitees zur Verhütung von Blindheit fand im April 2019 statt. In diesem Zeitraum wurden 993 Patienten untersucht und insgesamt 212 operative Eingriffe durchgeführt, davon $161 \mathrm{Ka}$ taraktoperationen. Beteiligt war medizinisches Personal aus Deutschland, der Schweiz und Tansania. 


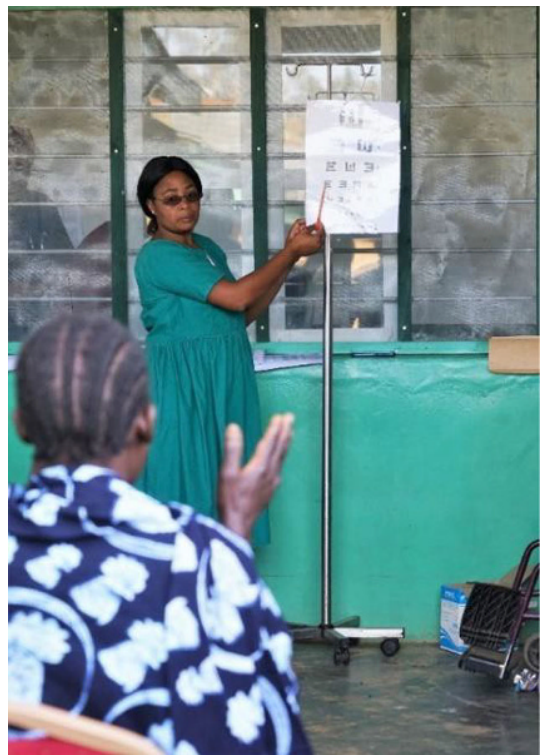

Abb. 1 ॥ Sehtest im Augencamp

Alle Patienten, die einer Kataraktoperationen zugeführt wurden, wurden zuvor in einem EDV-basierten System mit Namen, Geburtsdatum und Geschlecht erfasst. Die Anamnese erfolgte mittels eines kisuaheli/englisch sprechenden Krankenhausmitarbeiters. Es folgten im Weiteren Sehtest, Untersuchung an einer Spaltlampe und das Prüfen der Lichtreaktion.

Der Sehtest wurde jeweils prä- und postoperativ von einer "opthalmic nurse“ auf einer 5-m-Strecke mit E-Haken als „presenting visual acuity“ (PVA) vorgenommen: also Patienten, die keine Brille haben ohne Brille, bei Vorhandensein einer Brille auch mit Sehhilfe, postoperativ immer ohne (• Abb. 1).

Eine Kataraktoperation wurde nur bei vorhandener Lichtreaktion durchgeführt. Messung des Augendruckes oder Fundoskopie konnten aufgrund der eingeschränkten Ausstattung nicht routinemäßig durchgeführt werden.

In die Studie wurden nur Patienten ohne vorhergehende okuläre Traumata aufgenommen. Andere Begleiterkrankungen wie Glaukom, Pseudoexfoliationssyndrom, Vernarbungen der Hornhaut wurden nicht ausgeschlossen. Alle Patienten hatten zum Zeitpunkt der Operation eine mature Katarakt. Sie Sehschärfe betrug weniger als 6/60.

Präoperativ wurde bei allen Patienten eine Keratometrie mit einem $\mathrm{Ni}$ - dek ARK-1-Autorefraktionskeratometer (Nidek, Tokio, Japan) durchgeführt. Außerdem eine Achslängenmessung im Ultraschall-A-Mode mit einem Tomey AL-100-Biometriegerät (Tomey, Nürnberg, Deutschland).

Da der Schwerpunkt des Camps v. a. auch in der Ausbildung der tansanischen Kolleginnen und Kollegen bestand, wurden diese beiden Untersuchungen überwiegend von qualifizierten tansanischen Mitarbeitern („eye nurses“) unter Anleitung der deutschen Kollegen durchgeführt.

Die Linsenkalkulation erfolgte mittels der SKR-T-Formel mit einer Zielrefraktion für die jeweils operierten Augen von $-0,5 \mathrm{dpt}$.

Alle Patienten wurden nach unter Zeugen erfolgter mündlicher Einwilligung in der ortsüblichen „manual small incision cataract surgery“ (MSICS) mit Peribulbäranästhesie operiert [15]: Nach Anschlingen der M. rectus superior und Eröffnen der Bindehaut wurde ein ca. 6-7 mm großer sklerokornealer Tunnel bei 12 Uhr präpariert. Die Kapseleröffnung erfolgte mittels Can-openerTechnik. Nach Linsenentfernung wurde entsprechend der Biometrie eine faltbare Acryllinse (Acryl IOL Basis K, 1stQ, Mannheim) unter Eingabe von Viskoelastikum in den Kapselsack implantiert. Die Implantation der Intraokularlinsen erfolgte manuell und ungefaltet durch den sklerokornealen Tunnel. Das Absaugen von Rinde und Viskoelastikum nach der Linsenimplantation erfolgte mithilfe einer Simco-Kanüle. Zum Operationsende wurde das Auge mit balancierter Salzlösung (BSS) tonisiert und die Bindehaut über dem sklerokornealen Zugang mit Dexamethason unterspritzt. Alle in der Studie ausgewerteten MSICS-Operationen wurden durch 2 mit der Technik vertrauten tansanischen Kataraktchirurgen durchgeführt.

Eine obligatorische Kontrolle mit Visus und Spaltlampenmikroskopie erfolgte für alle operierten Patienten am Tag nach der Operation.

Die in Woche 1 operierten Patienten wurden gebeten, sich in Woche 2 des Camps zu einer zweiten postoperativen Kontrolle vorzustellen. Es wurde erneut eine Messung der Sehschärfe (PVA) durchgeführt, außerdem erfolgten eine Untersuchung an der Spaltlampe und eine Vermessung des operierten Auges mit dem Autorefraktometer.

Die Untersuchungsergebnisse wurden auf einem Untersuchungsbogen erfasst, in die EDV übertragen und später ausgewertet.

Soweit möglich wurden bei der Auswertung die Anforderungen der Deklaration von Helsinki in der aktuellen Fassung beachtet und eingehalten.

\section{Ergebnisse}

Von 161 operierten Kataraktpatienten kamen 42 Patienten zu einer weiteren postoperativen Kontrolle innerhalb des definierten Zeitfensters.

Das mittlere Kontrollintervall betrug 7 Tage, mindestens 5 und maximal 9 Tage.

Es konnte in jedem Fall die kalkulierte Intraokularlinse (IOL) implantiert werden. Die mittlere Stärke betrug 22,0 dpt. Die kleinste implantierte Linsenstärke war $17 \mathrm{dpt}$, die stärkste Linse hatte eine Brechkraft von $26 \mathrm{dpt}$.

Die mittlere postoperative Sehschärfe lag zum Zeitpunkt der zweiten Kontrolle bei 0,26 „presenting visual acuity“ (PVA), also unkorrigierte Sehschärfe auf der 5-m-Sehstrecke. Das schlechteste Ergebnis war Handbewegung, das beste Ergebnis 0,67.

Der mittlere Brechfehler (sphärisches Äquivalent) war $-2,82 \mathrm{dpt}$, Minimum $-7,25 \mathrm{dpt}$, Maximum 0,625 dpt.

Der Astigmatismus betrug im Mittel $-2,2$ dpt und variierte von $-5,75$ bis $-0,25 \mathrm{dpt}$. Die mittlere Achslage der Hornhautverkrümmung lag bei 113 Grad, was einem Astigmatismus gegen die Regel entspricht (• Tab. 1).

Die Weltgesundheitsorganisation hat Zielvorgaben für die Sehschärfe nach Kataraktoperation formuliert. Diese Zielvorgaben werden für die bestkorrigierte (BCVA) und unkorrigierte (UCVA) Sehschärfe unterschiedlich angegeben; $80 \%$ der operierten Patienten sollten eine unkorrigierte Sehschärfe von mehr als 0,3 erreichen - in unserem Kollektiv $14,2 \%$. Idealerweise haben nur $15 \%$ der operierten Patienten einen postoperativen Visus von $0,1-0,3$ und $5 \%$ von kleiner 0,1. Bei den von uns nachun- 
Ophthalmologe 2020 · 117:671-676 https://doi.org/10.1007/s00347-019-00983-9

(c) Der/die Autor(en) 2019

\section{S. Irle · E. Msigomba $\cdot$ K. Paust}

\section{Sumbawanga Augencamp follow-up Studie 2019}

\section{Zusammenfassung}

Hintergrund. Im Jahr 2019 hat das deutsche Komitee zur Verhütung von Blindheit (DKVB) ein Augencamp in der tansanischen Stadt Sumbawanga durchgeführt. Bei Patienten mit maturer Katarakt und intakter Lichtscheinwahrnehmung wurden Katarakte als „,manual small incision cataract surgery" (MSICS) operiert. Erstmalig wurde bei diesem Camp die Ergebnisqualität der durchgeführten Kataraktoperationen gemessen.

Ziel der Arbeit. Ziel war es, die Qualität der durchgeführten Kataraktoperationen darzustellen und die Ergebnisse in Zusammenhang mit den Vorgaben der Weltgesundheitsorganisation (WHO) zu bewerten.

Methoden. Patienten, die in den ersten Tagen des Augencamps kataraktoperiert worden waren, wurden in der zweiten Woche im Hinblick auf die Parameter Visus, Refraktion, spaltlampenmikroskopischer Befund und Komplikationen nachuntersucht, die Daten retrospektiv ausgewertet. Die Ergebnisse wurden mit den Vorgaben der WHO verglichen.

Ergebnisse. Es konnten 42 Patienten des Augencamps nach 5 bis 9 Tagen nachuntersucht werden. Folgende Parameter wurden gefunden: mittlere postoperative Sehschärfe 0,26 , sphärisches Äquivalent $-2,82 \mathrm{dpt}$, Astigmatismus $-2,2 \mathrm{dpt} / 113 \mathrm{Grad}$; Visus $>0,3$ in $14,2 \%$ (WHO $80 \%$ ), Visus $0,1-0,362 \%$ (WHO 15\%), Visus <0,1 in 23,8\% (WHO 5\%); verzögerter Heilungsverlauf in $29 \%$ der Fälle. Schlussfolgerung. Obwohl es zu einer Besserung des Sehvermögens kam, sind die
Ergebnisse ernüchternd im Vergleich zu den Vorgaben der WHO. Postoperativ zeigen sich ein myoper Shift und ein Astigmatismus gegen die Regel. Die Gründe sind: okuläre Komorbiditäten, eingeschränkte diagnostische und therapeutische Möglichkeiten, Ausbildungscharakter des Camps, erschwerte Rahmenbedingungen und fortgeschrittene Befunde. Die Ergebnisse der Studie sind wichtig, um die Qualität der eigenen Arbeit einschätzen zu können und um das Potenzial für künftige Verbesserungen ausloten zu können.

\section{Schlüsselwörter}

MSICS · Kataraktblindheit · Kataraktchirurgie Tansania $\cdot$ Sehschärfe

\section{Sumbawanga eye camp follow-up study 2019}

\section{Abstract}

Background. In 2019 the German Commission for the Prevention of Blindness (DKVB) held an eye camp in the Tanzanian town of Sumbawanga. For patients with mature cataracts and the ability to see light cateracts were treated by manual small incision cataract surgery (MSICS). For the first time in this camp the quality of the results of the cataract operations was measured.

Objective. The quality of the cataract operations is presented and the results were assessed in the context of the guidelines of the World Health Organization (WHO).

Methods. Those patients who had a cataract operation in the first week were examined during the second week with respect to the parameters vision, refraction, split lamp microscopic findings, results and complications after surgery. The results were retrospectively evaluated and compared with the guidelines of the WHO.

Results. A total of 42 postoperative patients from the eye camp could be examined within 5-9 days after cataract sugery. The following parameters were found: median postoperative visual acuity 0.26 , spherical equivalent $-2.82 \mathrm{dpt}$, astigmatism $-2.2 \mathrm{dpt}$, axis $113^{\circ}$. Visual acuity $>0.3$ in $14.2 \%$ (WHO $80 \%$ ), vision $0.1-0.3$ in $62 \%$ (WHO 15\%), vision $<0.1$ in $23.8 \%$ (WHO 5\%). Prolonged healing time and intraocular irritation in $29 \%$ of the cases. Conclusion. Although the vision improved, the results are sobering when taken in the context of the WHO guidelines. The postope- rative refraction showed a myopic shift and an high level of astigmatism. The reasons are manifold: ocular comorbidities, limited diagnostic and therapeutic possibilities in a nonclinical setting. Other factors are the kind of training of the staff in the camp, the difficult circumstances and advanced findings. Consideration of the results of this study is imperative to be able to measure the quality of the work and to create the potential to make future improvements.

\section{Keywords}

MSICS - Cataract blindness - Cataract surgery . Tanzania $\cdot$ Visual acuity tersuchten Patienten hatten $62 \%$ eine Sehschärfe zwischen 0,1 und 0,3 und $23,8 \%$ eine Sehschärfe unterhalb von 0,1 (• Tab. 2).

Bei der ausgewerteten postoperativen Kontrolle zeigte sich am 5. bis 9. Tag bei $71 \%$ der Patienten ein regulärer postoperativer Befund. Insgesamt $29 \%$ der Patienten hatten Auffälligkeiten: v. a. vermehrte Descemet-Falten, aber auch in 2 Fällen Fibrin in der Vorderkammer und bei 1 Patienten ein Glaskörperstrang vor der Linse (•Tab. 3 ).

\section{Diskussion}

Vorangestellt werden muss dem Diskussionsteil wohl die Frage, warum denn überhaupt Kataraktchirurgie in Form eines Augencamps und damit losgelöst von einer etablierten klinischen Versorgungsstruktur durchgeführt wird [2,9].

Vergleichbare Untersuchungen, von denen in der Literatur berichtet wird, wurden im Regelfall in Augenkliniken erhoben mit festen Strukturen im
Hinblick auf räumliche und personelle Ressourcen [5, 6, 13, 16-18].

An dieser Stelle sind 2 Antworten zu geben: Eines der nur teilweise gelösten Probleme ist in der Sub-Sahara-AfrikaRegion die Durchdringung der Bevölkerung mit der benötigten und nun auch zur Verfügung stehenden medizinischen Leistung. Es gibt eine Vielzahl von Barrieren, warum Menschen trotz Blindheit die Möglichkeit einer Kataraktoperation nicht Anspruch nehmen: Alter, fehlende finanzielle Mittel, keine Transportmög- 
Tab. 1 Postoperative Ergebnisse DKVB Sumbawanga Eye Camp 2019

\begin{tabular}{|c|c|c|c|}
\hline \multicolumn{4}{|c|}{ Ergebnisse der 42 nachkontrollierten Patienten } \\
\hline Parameter & Mittelwert & Minimum & Maximum \\
\hline Zeitintervall OP - zweite Post-OP-Kontrolle & 7 & 5 & 9 \\
\hline IOL Power (dpt) kalkuliert und implantiert & 22 & 17 & 26 \\
\hline Visus postoperativ (PVA) & 0,26 & Handbewegungen & 0,67 \\
\hline $\begin{array}{l}\text { Refraktion postoperativ sphärisches Äquiva- } \\
\text { lent (SE), (dpt) }\end{array}$ & $-2,82$ & $-7,25$ & 0,625 \\
\hline Astigmatismus postoperativ (dpt) & $-2,2$ & $-5,75$ & $-0,25$ \\
\hline Astigmatismus postoperativ Achslage $\left({ }^{\circ}\right)$ & 113 & - & - \\
\hline
\end{tabular}

Tab. 2 Postoperative Sehschärfe entsprechend WHO-Definition

\begin{tabular}{|c|c|c|c|}
\hline & $\begin{array}{l}\text { Good = Visus } \\
>0,3\end{array}$ & $\begin{array}{l}\text { Borderline = Visus } \\
0,1-0,3\end{array}$ & $\begin{array}{l}\text { Poor }=\text { Visus } \\
<0,1\end{array}$ \\
\hline WHO-Kriterien (\%) & 80 & 15 & 5 \\
\hline $\begin{array}{l}\text { Ergebnisse } \\
\text { Sumbawanga Eye Camp } 2019 \text { (\%) }\end{array}$ & 14,2 & 62 & 23,8 \\
\hline
\end{tabular}

Tab. 3 Dokumentierte Komplikationen bei der zweiten postoperativen Kontrolle

\begin{tabular}{ll} 
Art der Komplikation & Prozentualer Anteil (\%) \\
\hline $\begin{array}{l}\text { Komplikationen insgesamt } \\
\text { (Teilweise Mehrfachnennung) }\end{array}$ & 29 \\
$\begin{array}{l}\text { Descemet-Falten } \\
\text { Vermehrter Zellbefund in Vorderkammer }\end{array}$ & 14 \\
Fibrin & 2 \\
Glaskörperstrang in Vorderkammer & 5 \\
Pigment in Vorderkammer & 2 \\
Hyphäma & 7 \\
\hline
\end{tabular}

lichkeit, Skepsis gegenüber dem Verfahren ... $[9-12,19]$.

Es benötigt komplexe Programme, um das, was erwiesenermaßen gut ist, zu den Menschen zu bringen. Ein Augencamp ist einer der möglichen Lösungsansätze: Wenn die Menschen nicht zum Augenarzt kommen, kommt der Augenarzt zu den Menschen ...

Der zweite Grund für die Konzeption als Camp liegt in diesem Projekt darin, dass ein wesentliches Ziel der Arbeit in Sumbawanga die Ausbildung tansanischer Mitarbeiter zu Fachkräften in der augenmedizinischen Versorgung ist. Insofern ist das in Sumbawanga durchgeführte Camp ein Teilschritt hin auf dem Weg zu einer etablierten Versorgungsstruktur vor Ort. Bis dahin muss wohl in Kauf genommen werden, dass die Ergebnisse in etablierten Strukturen besser sind als in ländlichen $[2,10]$.
Es konnten 42 von 161 kataraktoperierten Augen (26\%) nachkontrolliert werden.

Die mittlere Nachbeobachtungszeit bis zur zweiten postoperativen Kontrolle betrug 7,2 Tage.

Das relativ kurze Kontrollintervall resultiert aus der Struktur des nur 2-wöchigen Camps. Ein Mindestintervall von 5 Tagen zwischen Operation und zweiter Kontrolluntersuchung wurde festgelegt, um eine relative Refraktionsstabilität und Reproduzierbarkeit bei der Auswertung der Daten zu erzielen; ebenso die Zahl der in die Studie eingeschlossenen Patienten im Verhältnis zur Zahl der operierten $\mathrm{Pa}$ tienten. Da in beiden Wochen operiert wurde, prinzipiell aber nur Patienten aus der ersten Woche nachkontrolliert werden konnten, sind nur $26 \%$ der operierten Patienten eingeschlossen worden. Eine gewisse Fluktuation liegt zum Teil vermutlich auch in den weiten Anreisewegen der Patienten wegen der überregionalen Bedeutung des Camps begründet.

Die Sehschärfe als „presenting visual acuity" (PVA) entspricht der Lebenssituation vieler operierter Patienten. Oft ist eine Brille für die Patienten nicht verfügbar oder bezahlbar. Insofern ist dieser Wert, obwohl in anderen Teilen der Welt unüblich, ein sinnvoller Parameter zur Darstellung der Ergebnisse aus Sicht des Patienten in seinem Umfeld $[5,18]$.

Die gemittelte Sehschärfe für die Ferne von nur 0,26 hängt mit mehreren Faktoren zusammen.

Okuläre Komorbiditäten wurden aufgrund der beschränkten diagnostischen und sprachlichen Möglichkeiten nicht ausgeschlossen; $100 \%$ der operierten $\mathrm{Au}$ gen hatten zum Operationszeitpunkt eine mature Linse. Fortgeschrittene Glaukome, traumatische Schäden am N. opticus und retinale Vorerkrankungen konnten im Vorfeld nicht ausgeschlossen werden und haben die Ergebnisqualität mit beeinflusst.

Wesentlicher Grund für das Ergebnis ist aber die deutliche myope postoperative Refraktion in Verbindung mit der hohen Hornhautverkrümmung.

Obwohl eine moderate Myopie besonders in Verbindung mit Emmetropie am Partnerauge im Sinne einer Monovision akzeptabel, sogar wünschenswert wäre, sind hier besondere Anstrengungen nötig, um die Ergebnisqualität zu verbessern.

Natürlich ist bei der Vermessung des Auges, Auswahl der Intraokularlinse und Art der Operation ein Verfahren zu wählen, das die Notwendigkeit für das Tragen einer Brille nach dem operativen Eingriff minimiert $[1,14,22]$.

Schlüsselparameter für die Wahl der optimalen Intraokularlinse sind Keratometrie und Biometrie. Auch kleine Fehler bei diesen diagnostischen Verfahren führen $\mathrm{zu}$ erheblichen Fehlrefraktionen postoperativ [2, 13, 22]. In Verbindung mit dem Ausbildungscharakter des Camps und in Anbetracht der ausschließlich maturen Ausgangssituationen mit fehlendem Fixationsvermögen bei den Achslängenmessungen und der Keratometrie ist hier offensichtlich ein deutlicher myoper Shift entstanden. 
Einfluss haben sicher auch die voreingestellte myope Zielrefraktion von $-0,5 \mathrm{dpt}$, ein $\mathrm{zu}$ hoher Anpressdruck mit der Biometriesonde, das kurze postoperative Kontrollintervall, der Verbleib von Resten des Viskoelastikums hinter der IOL und die Tatsache, dass doch ein größerer Teil der Intraokularlinsen mutmaßlich nicht, wie beabsichtigt, in den Kapselsack, sondern in den Sulcus ciliaris implantiert wurde. Ein einfacherer Implantationsvorgang und eine bessere Positionierung der IOL wären vermutlich auch mit nichtfaltbaren PMMA-Linsen $\mathrm{zu}$ erzielen gewesen, diese standen aber nicht zur Verfügung.

Interessanterweise konnte Sherwin et al. bei Kataraktoperationen mit einer +22,0-dpt-Standardintraokularlinse in Malawi deutlich bessere Ergebnisse erzielen, als das in Sumbawanga möglich war [17].

Die gemittelte Brechkraft der implantierten Linsen lag auch in Sumbawanga bei 22,0 dpt. Trotzdem war die postoperative Sehschärfe nicht auf gleichem Niveau wie in Malawi. Grund dafür könnte der zweite elementare refraktive Fehler sein, der hohe Astigmatismus.

In einer Studie aus den 60er-Jahren an Schülern aus der Region zeigt sich in der Population kein höherer Astigmatismus als üblich [7]. Dennoch betrug der postoperative Astigmatismus -2,2 dpt mit einer Achse von 113 Grad. Obwohl auch andere Autoren von ähnlichen Ergebnissen nach MSICS berichten [22], gibt es durchaus auch zahlreiche Artikel, die besonders im Vergleich zur Kataraktoperation mit Phakoemulsifikation darlegen, dass MSICS nicht zwingend $\mathrm{zu}$ höheren postoperativen Astigmatismen führt $[4,6,10,16,19]$. Insofern sind die in Sumbawanga gemessenen Astigmatismen durchaus eine Anfrage an die spezielle Technik der Tunnelpräparation und weisen hin auf Verbesserungspotenzial. Verschiedene Techniken sind in diesem Zusammenhang beschrieben, die geeignet sind, die Höhe der operativ induzierten Hornhautverkrümmung zu reduzieren oder Einfluss auf die Achslage zu nehmen [15].

Wenn das Verbesserungspotenzial komplexer Prozesse ausgeschöpft werden soll, erfordert dass die Kenntnis der eigenen Qualitätsparameter sowie ein gutes Komplikations- und Fehlermanagement. Einer der Qualitätsparameter ist dabei die Zahl der Kataraktoperationen pro Operateur und Jahr [1, 14]. Beide Operateure des Sumbawanga Camps waren seit Jahren mit der MSICS-Technik vertraut.

Robert Lindfield schlägt ein Benchmarking-System vor, das es erlaubt, die eigenen Ergebnisse zu analysieren, um für die Zukunft neue Ziele zu formulieren, die wiederum in einen kontinuierlichen Verbesserungsprozess münden [13, 14].

Am deutlichsten wird das Optimierungspotenzial der Ergebnisse aus dem Sumbawanga Camp beim Vergleich mit den Zielvorgaben der Weltgesundheitsorganisation. Hilfreich bei der Einschätzung sind dabei allerdings die Statements des Vision 2020 Research Mentorship Workshop in Moshi, Tanzani, vom Januar 2017. Internationale Experten unter anderem aus Tansania, Uganda und Kenia stellten in einem Delphi Prozess fest, dass die Zielsetzungen der WHO in der Sub-Sahara-Afrika-Region schwierig zu erreichen sind. Ähnliches wird auch aus anderen Regionen der Welt berichtet, so Trinidad und Tobago, Philippinen und Bangladesch [1, 13, 18]. Offensichtlich gibt es eine nicht unerhebliche Diskrepanz zwischen den wünschenswerten Zielvorgaben und den in der Realität erreichbaren Ergebnissen.

Diese Einschätzung soll nicht das Bemühen reduzieren, Verbesserungspotenzial zu identifizieren und einmal erkannte Parameter auch systematisch nachzujustieren, um die Ergebnisqualität zu verbessern.

In dem oben zitierten Workshop wurden folgende Punkte genannt, um die Ergebnisse der Kataraktchirurgie in Afrika zu verbessern [1]:

1. verbessertes Training der Kataraktchirurgen,

2. Biometrie optimieren,

3. Ausstattung verbessern (Instrumente/Mikroskope),

4. Monitoring der Ergebnisqualität der Kataraktchirurgie durch den Operateur,
5. Ausbildung aller Teammitglieder, die am Katarakt-OP-Prozess beteiligt sind,

6. Monitoring der refraktiven Ergebnisse,

7. $\ldots$

Möglicherweise ist es sogar sinnvoll, solche Faktoren im Sinne eines „capacity building" sehr regelmäßig nachzuhalten, um gemeinsam Anreize zu schaffen, den Verbesserungsprozess in Gang zu halten [8].

Am Ende gilt wohl wie überall auf der Welt, dass eine überzeugende Ergebnisqualität langfristig das beste Argument für eine erfolgreiche Kataraktchirurgie ist [2]. Es bringt den betroffenen Menschen gar nichts, wenn man sie von der Kataraktblindheit in eine Refraktionsblindheit überführt.

Die an diesem Prozess Beteiligten auch im Rahmen eines Augencamp-Programmes - sind für die Qualität ihrer Ergebnisse verantwortlich.

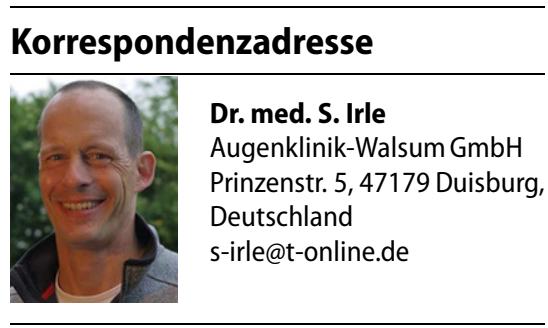

\section{Einhaltung ethischer Richtlinien}

Interessenkonflikt. S. Irle, E. Msigomba und K. Paust geben an, dass kein Interessenkonflikt besteht.

Für diesen Beitrag wurden von den Autoren keine Studien an Menschen oder Tieren durchgeführt. Für die aufgeführten Studien gelten die jeweils dort angegebenen ethischen Richtlinien.

Open Access. Dieser Artikel wird unter der Creative Commons Namensnennung 4.0 International Lizenz (http://creativecommons.org/licenses/by/4.0/deed. de) veröffentlicht, welche die Nutzung, Vervielfältigung, Bearbeitung, Verbreitung und Wiedergabe in jeglichem Medium und Format erlaubt, sofern Sie den/die ursprünglichen Autor(en) und die Quelle ordnungsgemäßnennen, einen Link zur Creative Commons Lizenz beifügen und angeben, ob Änderungen vorgenommen wurden. 


\section{Literatur}

1. Buchan JC et al (2018) What are the priorities for improving cataract surgical outcomes in Africa? Results of a Delphi exercise. Int Ophthalmol 38:1409-1414

2. Chang MA et al (2008) The surgical management of cataract: barriers, best practices and outcomes. Int Ophthalmol 28:247-260

3. Comm Eye Health (Hrsg) (2018) Cataract surgical rates 30: 88-89. https://www.cehjournal.org/ article/cataract-surgical-rates. Zugegriffen: 20 . Juni 2019

4. Fasina $O$ et al (2017) Cataracat surgery in Ibadan, Nigeria: visual outcome and postoperative refractive error. Ann Health Res 3:43-49

5. Isawumi MA et al (2009) Evaluation of cataract surgery outcome in western Nigeria. Ghana Med J 43:169-174

6. Jha KN (2006) Manual small incision cataract surgery: experience at a military hospital. Med J Armed Forces India 62:212-215

7. Johnstone WW, McLaren DS (1963) Refraction anomalies in tanganyikan children. Br J Ophthalmol 47:95-108
8. Judson Ket al (2017) Impact of systematic capacity building on cataract surgical service development in 25 hospitals. BMC Ophthalmol 17:96

9. Kessy JP, Lewallen S (2007) Poverty as a barrier to accessing cataract surgery: a study from Tanzania. BrJOphthalmol 91:1114-1116

10. Khanna R et al (2011) Cataract surgery in developing countries. Curr Opin Ophthalmo 22:10-14

11. Lewallen S et al (2005) Increasing cataract surgery to meet Vision 2020 targets; experience from tho rural programmes in east Africa. Br J Ophthalmol 89:1237-1240

12. Lewallen $\mathrm{S}$ et al (2006) Willingness to pay for cataract surgery in two regions of Tanazania. Br J Ophthalmol 90:11-13

13. Lindfield $R$ et al (2009) Outcome of cataract surgery at one year in Kenya, the Philippines and Bangladesh. Br JOphthalmol 93:875-880

14. Lindfield R (2014) Improving the quality of cataract surgery. Community Eye Health 27(85):9-11

15. Natchiar G (2012) Manual small incision cataract surgery. Aravind eye hospital

16. Semanyenzi SE (2015) Outcome after small incision cataract surgery (SICS) and phacoemulsification at Kigali University Teaching Hospital. Rwanda Medical Journal 72(4):12-16
17. Sherwin JC et al (2012) Outcomes of manual smallincision catarat surgery using standard 22 dioptre intraocular lenses at Nkhoma Eye Hospital, Malawi. Int Ophthalmol 32:341-347

18. Sonron EA et al (2015) A retrospective study on the outcomes of cataract surgery in an Eastern Regional Health Authority hospital of Trinidad and Tobago. PeerJ 3:e1222. https://doi.org/10.7717/ peerj.1222

19. Tabin $\mathrm{G}$ et al (2008) Cataract surgery for the developing world. Curr Opin Ophthalmol 19:55-59

20. World Health Assembly, World Health Organization (2013) Draft action plan for the prevention of avoidable blindness and visual impairment 2014-2019. Towards universal eye health: a global action plan 2014-2019 (WHA66/11). http:// apps.who.int/gb/ebwha/pdf_files/WHA66/A66 11-en.pdf. Zugegriffen: 20. Juni 2019 (Informal consultation on analysis of blindness prevention outcomes. Geneva. WHO. WHO/PBL/98/68)

21. Wong TY (2015) Cataract surgery programmes in Africa. Br JOphthalmol 89:1231-1232

22. Yorston D (2002) Does prospecitve monitoring improve cataract surgery outcomes in Africa? Br J Ophthalmol 86:543-547

\section{Praxis-Finanzrechner für Ärzte Neues Tool auf SpringerMedizin.de}

Wie rentabel läuft meine Praxis? Wie stehe ich im Vergleich zu meinen Kollegen im Bundesland? Welche Anschaffungen kann ich mir leisten? Antworten auf diese betriebswirtschaftlichen Fragen liefert künftig ein Tool auf SpringerMedizin.de, indem es aktuelle Versorgungs- und Abrechnungsdaten sowie Analysedaten deutscher Gesundheitsunternehmen zu Grunde legt.

Mit den kostenfreien Praxis-Finanzrechnern können sich vor allem in der Praxis tätigen Ärzte, die sich oft auch unternehmerischen Herausforderungen stellen müssen, interaktiv mit den Themen Controlling und Benchmarking auseinandersetzen:

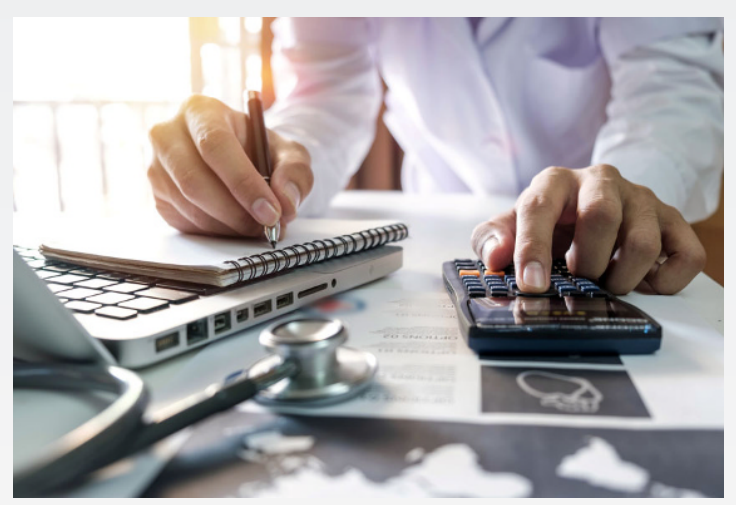

(c) mrmohock/stock.adobe.com
> Mit dem Controlling-Assistenten behalten Sie die wichtigsten wirtschaftlichen Praxiskennzahlen immer im Auge. $>$ Mit dem Tool Benchmark können Sie Ihre Praxis mit anderen Praxen vergleichen.

> Ihren Praxisstandort - ebenfalls ein wichtiger Faktor für den Erfolg - analysieren Sie mit Standortcheck aus verschiedenen Perspektiven

> Mit dem Investitionsassistenten können sie sehen, ob und wann sich eine Geräteinvestition amortisiert und Sie bekommen auch Anhaltspunkte, welche Einnahmen Sie zu erwarten haben.

Die Praxis-Tools sind ein externes Angebot von Rebmann Research, Partner von SpringerMedizin.de. Sie finden die Praxis-Finanzrechner auf SpringerMedizin.de unter dem Navigationspunkt "Mehr".

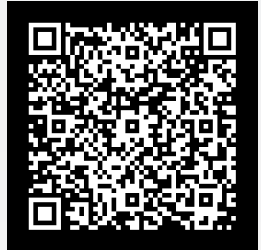

